\title{
Assessment of drug utilization pattern and risk factors for the development of diabetic neuropathy among type 2 diabetic patients in a south Indian hospital: A cross-sectional observational study
}

Lavanya Nalluri ${ }^{1}$, Madhavi Mannam ${ }^{1}$, Navya Vemireddy ${ }^{1}$, Nandini Akula ${ }^{1}$, Mounika Pothuraju${ }^{1}$, Dhanalakshmi Pinnika ${ }^{1}$, Jaidev Sudagani ${ }^{2}$, Ravindrababu Pingili ${ }^{3}$ Naveen Babu Kilaru ${ }^{4 *}$

${ }^{1}$ Department of Pharmacy Practice, KVSR Siddhartha College of Pharmaceutical Sciences, Vijayawada, India.

${ }^{2}$ Endocrinologist, Santhi Endocrine and Diabetes Hospital, Vijayawada, Andhra Pradesh, India.

${ }^{3}$ Department of Pharmacology, KVSR Siddhartha College of Pharmaceutical Sciences, Vijayawada, India.

${ }^{4}$ Department of Pharmaceutical Biotechnology, KVSR Siddhartha College of Pharmaceutical Sciences, Vijayawada, India.

\begin{tabular}{l}
\hline ARTICLE INFO \\
\hline Received on: 12/04/2019 \\
Accepted on: $28 / 09 / 2019$ \\
Available online: 03/12/2019 \\
\hline Key words: \\
Diabetic neuropathy, \\
prevalence, hyperglycemia, \\
odds ratio.
\end{tabular}

odds ratio.

\begin{abstract}
Diabetic peripheral neuropathy (DPN) is a well-known microvascular complication of type 2 diabetes mellitus (T2DM). DPN is defined as peripheral nerve dysfunction in diabetics after exclusion of other causes. To assess the prevalence of peripheral neuropathy in T2DM and the associated risk factors among in outpatients department in a south Indian hospital. A cross-sectional observational study was conducted on 868 subjects (509 with DPN and 359 without DPN). Prevalence of diabetic peripheral neuropathy was measured and risk factors for the development of diabetic peripheral neuropathy were determined by calculating odds ratios and drug utilization pattern was assessed. The prevalence of DPN in T2DM was significantly higher in the subjects who are married, uneducated, housewives, and urban residents. Many associated risk factors could affect T2DM leading to DPN such as hypertension, other diseases, endocrine diseases, history of cardiovascular diseases (CVD), $>9 \mathrm{HbAlc}$, low high-density lipoproteins (HDL), high serum creatinine, long duration of diabetes, physical inactivity, and habit of taking junk foods (weekly once and weekly twice, soft drinks occasionally). The present study revealed that risk factors for the development of DPN were hypertension, endocrine diseases, history of CVD, poor glycemic control ( $>9 \mathrm{HbA1c}$ ), low HDL, high serum creatinine, long duration of diabetes, physical inactivity, habit of taking junk foods and soft drinks. Early detection of the identification of DPN in T2DM is needed in order to slow progression and complications. Metformin (40.47\%), combination of glimepiride and metformin (29.93\%), combination of human insulin and insulin isophane $(22.7 \%)$ were mostly given to the T2DM patients with neuropathy.
\end{abstract}

\section{INTRODUCTION}

With 387 million people diagnosed with diabetes mellitus worldwide and a prevalence of $8.2 \%$ as per the Diabetes atlas 2014 (Joaoda et al., 2016). Diabetic peripheral neuropathy (DPN) is a well-known microvascular complication of type 2 diabetes mellitus (T2DM) and is defined as peripheral nerve

\footnotetext{
${ }^{*}$ Corresponding Author

Naveen Babu Kilaru, Department of Pharmaceutical Biotechnology, KVSR Siddhartha College of Pharmaceutical Sciences, Vijayawada, Andhra Pradesh, India.E-mail: naveenbabukvsr@gmail.com
}

dysfunction in diabetics (Boulton et al., 1998; Candrilli et al., 2007; Sumner et al., 2003; William and Laurence, 2005). Some of the risk factors of DPN include age $>60$ years, females, obesity, and hypertension (Bruce et al., 2008). Regular consumption of even a moderate amount of alcohol interferes with blood glucose and increases the risk of peripheral neuropathy (Emanuele et al., 1998). Similarly, smoking and long duration of diabetes mellitus are found to increase the risk of DPN. One hundred and ten million people worldwide are estimated to be likely affected by DPN (Tesfaye, 2004). The prevalence of DPN varies largely across regions from 5\% to 60\% (Davies et al., 2006; Tesfaye and Selvarajah, 2012; Tesfaye et al., 1996; Young et al., 1993). 
The World Health Organization defines "drug utilization" as the marketing, distribution, prescription, and use of the drugs in a society considering its medical, social, and economic consequences (Amit et al., 2017). Drug utilization studies help to assess whether the drug treatment is rational or not and to determine rational drug use especially in poorer and rural populations (Sekhar et al., 2016). The objective of the present study was to assess the drug utilization pattern, prevalence, and risk factors which are responsible for the development of DPN and to assess the drug utilization pattern.

\section{METHODS}

A cross-sectional observational study was conducted on patients with or without T2DM at outpatients department of Dr. Pinnamaneni Siddhartha Institute of Medical Sciences, Vijayawada, Andhra Pradesh, India from Dec 1, 2017 to May 31, 2018. The study protocol was approved by the Ethical Committee of KVSR Siddhartha College of Pharmaceutical Sciences (SCOPS), Vijayawada, India with KVSRSCOPS/IEC/ PG/231/2017 approval number.

\section{Participants selection}

Patients with or without T2DM complications of both sexes and agreeable to take part in the planned study was included. A total of 868 patients (359 patients with T2DM and 509 patients with DPN) were enrolled in the study.

\section{Inclusion criteria}

Patients who are visiting the public endocrine hospital in the duration of 6 months would be recruited.

\section{Exclusion criteria}

Patients with incomplete case reports, gestational, type 1 , and maturity onset diabetes were excluded.

\section{Data collection}

Biochemical, clinical data, demographics, socioeconomic (using modified Kuppuswamy's scale), and lifestyle characteristics were collected with the prior consent of the participant. DPN was assessed using sensations of a pinprick, reflexes of ankles, and vibration perception threshold test. Data were collected from a total of 868 patients (359 patients with T2DM and 509 patients with DPN).

\section{Statistical analysis}

In the descriptive statistical analysis, categorical variables were expressed as numbers and percentages. For categorical variables, the tests of significance analysis, we applied a Chi-Square test using Graph Pad Prism Software (Version 5.0). $p<0.05$ was considered significant. Odds ratio with $95 \%$ confidence intervals was calculated using multivariate regression analysis using statistical package for the social sciences software.

\section{RESULTS}

A total of 868 subjects (359 with type 2 diabetes and 509 with DPN) data were presented in Table 1. Tables 2 and 3 show the socio demographics and life style characteristics of subjects with and without DPN, respectively. The prevalence of diabetic peripheral neuropathy was found to be $41.24 \%$. The prevalence of DPN was significantly higher in the subjects who are married (99.4\%, $p=0.0001)$, uneducated $(66.8 \%, p<0.0001)$, nature of work (housewives), urban residents $(61.5 \%, p=0.0048)$, co-morbidities like hypertension $(40.41 \%, p<0.0001)$, other diseases $(25.2 \%, p<0.0001)$, endocrine diseases $(11.64 \%, p=$ $0.0068)$, history of cardiovascular diseases $(8.21 \%, p<0.0001)$, no physical activity (62\%), habit of taking junk foods (weakly once $p<0.0001$, weakly twice $p=0.0010$ ), soft drinks (taking occasionally $26.4 \%, p=0.0010)$, HbAlc $(7 \%-9 \%,>9 \% p<$ $0.0001)$, low high-density lipoproteins (HDL) $(23 \%, p=0.0206)$, high serum creatinine $(14.6 \%, p<0.0001)$, duration of diabetes ( $>10$ years, 37.6\%, $p<0.0001 ; 5-10$ years, 35.4\%, $p<0.0001$ ). Gender, age, body mass index (BMI), body weight, monthly income, and blood glucose levels are not significantly associated with the development of DPN.

The odds ratios for risk factors in patients with T2DM and DPN were presented in Table 4 . The analysis showed that married (OR, 7.868; 95\% CI, 2.275-27.22, $p=0.0001$ ), poorly educated (OR, 0.2856; 95\% CI, 0.2151-0.3791, $p<0.0001)$, housewives (OR, 0.5254 ; 95\% CI, 0.3477-0.7938, $p=0.0021$ ), urban residents (OR, 0.6602; 95\% CI, 0.4944-0.8815, $p=$ 0.0048), hypertension (OR, 2.642; 95\% CI, 1.906-3.661, $p<$ 0.0001 ), other diseases (OR, 2.030; 95\% CI, 1.434-2.874, $p<$ 0.0001 ), Endocrine diseases (OR, 1.780; 95\% CI, 1.170-2.708, $p$ $=0.0068)$ history of cardiovascular diseases (CVD) (OR, 10.59; 95\% CI, 4.648-24.14, $p<0.0001$ ), HbA1c (OR, 2.001; 95\% CI, 1.432-2.796, $p<0.0001$ ), low HDL (OR, 0.5934; 95\% CI, $0.3807-0.9251, p=0.0206$ ), high serum creatinine (OR, 11.10; 95\% CI, 3.320-37.11, $p<0.0001)$, long duration of diabetes (OR, 3.155; 95\% CI, 2.228-4.468), physical inactivity(OR, $0.5874 ; 95 \%$ CI, $0.44670 .7723, p=0.0001)$, junk foods weekly once (OR, 2.732; 95\% CI, 1.729-4.319, $p<0.0001)$ and weekly twice (OR, 2.385; 95\% CI, 1.4102-4.036, $p=0.0010)$, and soft drinks occasionally (OR, 1.776; 95\% CI, 1.260-2.505, $p$ $=0.0010)$. Drug utilization pattern details were summarized in Table 5. Metformin, combination of glimepiride and metformin, combination of human insulin and insulin isophane were mostly given to the T2DM patients with DPN.

\section{DISCUSSION}

Prevalence, risk factors for DPN, and drug utilization pattern were assessed in an outpatients department at a tertiary care hospital. The present study results suggested that subjects who are married, uneducated, housewives, urban residents, and risk factors were co-morbidities (hypertension, history of cardiovascular diseases, endocrine diseases, and other diseases), no physical activity, habit of taking junk foods (weekly once, weekly twice), soft drinks (taking occasionally), poor glycemic control, low HDL, and serum creatinine are major risk factors for DPN. The results are consistent with the following studies.

The risk factors and prevalence of DPN among patients with T2DM were assessed by Nahla et al. (2018). The case of Jordan and concluded that marital status was one of the significant risk factors associated with the development of diabetic neuropathy (Nahla et al., 2018). In the current study also marital status $(99.4 \%, p=0.0001)$ was associated and was the major risk factor for DPN (OR, 7.868; 95\% CI, 2.275-27.22). 
Table 1. Biochemical and clinical characteristics of patients with type 2 diabetes mellitus $(N=359)$.

\begin{tabular}{|c|c|}
\hline Variable & $\begin{array}{c}\text { Patients with T2DM } \\
N(\%)\end{array}$ \\
\hline \multicolumn{2}{|l|}{ Gender } \\
\hline Male & $155(43.2)$ \\
\hline Female & $204(56.8)$ \\
\hline \multicolumn{2}{|l|}{ Age (Years) } \\
\hline $0-20$ years & $1(0.3)$ \\
\hline $21-40$ years & $83(23.2)$ \\
\hline $41-60$ years & $217(60.6)$ \\
\hline Above 60 years & $57(15.9)$ \\
\hline \multicolumn{2}{|l|}{ Marital status } \\
\hline Unmarried & $16(4.5)$ \\
\hline Married & $343(95.5)$ \\
\hline \multicolumn{2}{|l|}{ Education } \\
\hline Uneducated & $131(36.5)$ \\
\hline Educated & $228(63.5)$ \\
\hline \multicolumn{2}{|l|}{$\operatorname{BMI}\left(\mathrm{kg} / \mathrm{m}^{2}\right)$} \\
\hline$<25 \mathrm{~kg} / \mathrm{m}^{2}$ & $114(31.8)$ \\
\hline$\geq 25 \mathrm{~kg} / \mathrm{m}^{2}$ & $245(68.2)$ \\
\hline \multicolumn{2}{|l|}{ Body weight (kg) } \\
\hline$<50$ & $5(1.3)$ \\
\hline $50-70$ & $161(45)$ \\
\hline$>70$ & $192(53.6)$ \\
\hline \multicolumn{2}{|l|}{ Nature of work } \\
\hline Not working any where & $41(11.4)$ \\
\hline Private job & $93(25.9)$ \\
\hline Govt. job & $39(10.8)$ \\
\hline Daily labor & $38(10.6)$ \\
\hline Housewife & $148(41.3)$ \\
\hline \multicolumn{2}{|l|}{ Locality } \\
\hline Rural & $105(29.2)$ \\
\hline Urban & $254(70.7)$ \\
\hline \multicolumn{2}{|l|}{ Monthly income } \\
\hline No income & $170(47.5)$ \\
\hline Below 25,000 & $115(32.1)$ \\
\hline Above 25,000 & $73(20.4)$ \\
\hline \multicolumn{2}{|l|}{ Co-morbidities } \\
\hline No & $131(29.4)$ \\
\hline HTN & $138(30.8)$ \\
\hline History of CVDs & $7(1.56)$ \\
\hline Endocrine diseases & $59(13.2)$ \\
\hline Other diseases & $112(25.1)$ \\
\hline \multicolumn{2}{|l|}{ Systolic blood pressure } \\
\hline$<140 \mathrm{mmHg}$ & $259(72.1)$ \\
\hline$\geq 140 \mathrm{mmHg}$ & $100(27.9)$ \\
\hline \multicolumn{2}{|l|}{ Diastolic blood pressure } \\
\hline$<90 \mathrm{mmHg}$ & $281(78.3)$ \\
\hline$\geq 90 \mathrm{mmHg}$ & $78(21.7)$ \\
\hline
\end{tabular}

Table 1. (Continued)

\begin{tabular}{lr}
\hline Variable & $\begin{array}{r}\text { Patients with } \\
\boldsymbol{N}(\%)\end{array}$ \\
\hline HbA1C & \\
$<7$ & $141(44.2)$ \\
$7-9$ & $109(34.2)$ \\
$>9$ & $69(21.6)$ \\
Fasting blood glucose (mg/dl) & \\
$70-80$ & $10(3)$ \\
$80-120$ & $92(27.6)$ \\
$121-160$ & $107(32$ \\
$161-200$ & $71(21.3)$ \\
$>200$ & $54(16.2$ \\
Postprandial blood glucose & \\
levels (mg/dl) & $3(1)$ \\
$90-110$ & $9(3)$ \\
$111-130$ & $33(10.9)$ \\
$131-150$ & $165(54.6)$ \\
$151-200$ & $92(30.5)$ \\
$>200$ &
\end{tabular}

Random blood glucose (mg/dl)

$\begin{array}{lc}80-100 & 0 \\ 101-120 & 0 \\ 121-140 & 0 \\ 141-160 & 2(13.3) \\ 161-200 & 1(6.7) \\ >200 & 12(80)\end{array}$

HDL $(\mathrm{mg} / \mathrm{dl})$

Not available $\quad 54(20.1)$

Normal $130(48.3)$

Low $55(20.4)$

High 30 (11.2)

Triglycerides (mg/dl)

Not available $\quad 54(20.5)$

Normal $109(41.5)$

Low $8(3)$

High $92(35)$

Total cholesterol (mg/dl)

Not available $\quad 54(19.6)$

Normal $151(54.7)$

Low $6(2.2)$

High $65(23.6)$

LDL (mg/dl)

Not available $\quad 57(20.8)$

Normal $163(59.4)$

Low $9(3.3)$

High $\quad 45$ (16.5)

Urea $(\mathrm{mg} / \mathrm{dl})$

Not available $\quad 72(36.4)$

Normal 78 (39.4) 
Table 1. (Continued)

\begin{tabular}{lc}
\hline Variable & $\begin{array}{c}\text { Patients with T2DM } \\
\boldsymbol{N}(\%)\end{array}$ \\
\hline Low & 0 \\
High & $48(24.2)$ \\
Serum creatinine $(\mathrm{mg} / \mathrm{dl})$ & \\
Not available & $45(12.6)$ \\
Normal & $305(85.2)$ \\
Low & $5(1.4)$ \\
High $=$ & $3(0.8)$ \\
Duration of T2DM (years) & $172(47.9)$ \\
$<5$ & $111(30.9)$ \\
$5-10$ & $76(21.2)$ \\
$>10$ & \\
Following T2DM education & $282(79.2)$ \\
Yes & $74(20.8)$ \\
No & \\
\hline T2DM = type 2 diabetes mellitus, BMI = body mass index, HTN = hypertension, & \\
CVDs $=$ cardiovascular diseases, HbA1C = glycated hemoglobin, HDL = high- \\
density lipoproteins, LDL = low-density lipoproteins.
\end{tabular}

Table 2. Socio-demographic characteristics of diabetic patients with $(N=509)$ or without diabetic peripheral neuropathy $(N=359)$.

\begin{tabular}{|c|c|c|c|}
\hline Variable & $\begin{array}{c}\text { Patients with } \\
\text { T2DM } \\
N(\%)\end{array}$ & $\begin{array}{c}\text { Patients with } \\
\text { T2DM and DPN } \\
N(\%)\end{array}$ & $p$-Value \\
\hline \multicolumn{4}{|l|}{ Gender } \\
\hline Male & $155(43.2)$ & $199(39.1)$ & Ref \\
\hline Female & $204(56.8)$ & $310(60.9)$ & 0.2284 \\
\hline \multicolumn{4}{|l|}{ Age } \\
\hline $0-20$ years & $1(0.3)$ & - & Ref \\
\hline $21-40$ years & $83(23.2)$ & $41(8.1)$ & 0.4830 \\
\hline $41-60$ years & $217(60.6)$ & $318(62.5)$ & 0.2267 \\
\hline Above 60 years & $57(15.9)$ & $150(29.5)$ & 0.1070 \\
\hline \multicolumn{4}{|l|}{ Marital status } \\
\hline Unmarried & $16(4.5)$ & $3(0.6)$ & Ref \\
\hline Married & $343(95.5)$ & $506(99.4)$ & $0.0001^{* * * *}$ \\
\hline \multicolumn{4}{|l|}{ Education } \\
\hline Uneducated & $131(36.5)$ & $340(66.8)$ & Ref \\
\hline Educated & $228(63.5)$ & $169(33.2)$ & $<0.0001^{* * * *}$ \\
\hline \multicolumn{4}{|l|}{ BMI $\left(\mathrm{kg} / \mathrm{m}^{2}\right)$} \\
\hline$<25 \mathrm{~kg} / \mathrm{m}^{2}$ & $114(31.8)$ & $132(25.9)$ & Ref \\
\hline$\geq 25 \mathrm{~kg} / \mathrm{m}^{2}$ & $245(68.2)$ & $377(74.1)$ & 0.0609 \\
\hline \multicolumn{4}{|l|}{ Body weight (kg) } \\
\hline$<50$ & $5(1.3)$ & $14(2.8)$ & Ref \\
\hline $50-70$ & $161(45)$ & $240(47.3)$ & 0.2281 \\
\hline$>70$ & $192(53.7)$ & $254(50)$ & 0.1483 \\
\hline \multicolumn{4}{|l|}{ Nature of work } \\
\hline $\begin{array}{l}\text { Not working } \\
\text { anywhere }\end{array}$ & $41(11.4)$ & $116(22.8)$ & Ref \\
\hline Private job & $93(25.9)$ & $111(21.8)$ & $0.0001^{* * * *}$ \\
\hline Govt. job & $39(10.8)$ & $24(4.8)$ & $<0.0001^{* * *}$ \\
\hline
\end{tabular}

Table 2. (Continued)

\begin{tabular}{|c|c|c|c|}
\hline Variable & $\begin{array}{c}\text { Patients with } \\
\text { T2DM } \\
N(\%)\end{array}$ & $\begin{array}{c}\text { Patients with } \\
\text { T2DM and DPN } \\
N(\%)\end{array}$ & $p$-Value \\
\hline Daily labor & $38(10.6)$ & $38(7.5)$ & $0.0003^{* * * *}$ \\
\hline Housewife & $148(41.2)$ & $220(43.2)$ & $0.0021^{* *}$ \\
\hline \multicolumn{4}{|l|}{ Locality } \\
\hline Rural & $105(29.2)$ & $196(38.5)$ & Ref \\
\hline Urban & $254(70.8)$ & $313(61.5)$ & $0.0048^{* *}$ \\
\hline \multicolumn{4}{|l|}{ Monthly income } \\
\hline No income & $170(47.5)$ & $315(62)$ & Ref \\
\hline Below 25,000 & $115(32.1)$ & $159(31.3)$ & 0.0587 \\
\hline Above 25,000 & $73(20.4)$ & $34(6.6)$ & $<0.0001 * * *$ \\
\hline \multicolumn{4}{|l|}{ Co-morbidities } \\
\hline No & $131(29.4)$ & $106(14.52)$ & Ref \\
\hline HTN & $138(30.8)$ & $295(40.41)$ & $<0.0001^{* * * *}$ \\
\hline History of CVDs & $7(1.56)$ & $60(8.21)$ & $<0.0001^{* * * *}$ \\
\hline $\begin{array}{l}\text { Endocrine } \\
\text { diseases }\end{array}$ & $59(13.2)$ & $85(11.64)$ & $0.0068^{* *}$ \\
\hline Other diseases & $112(25.1)$ & $184(25.2)$ & $<0.0001^{* * * *}$ \\
\hline \multicolumn{4}{|l|}{$\begin{array}{l}\text { Systolic blood } \\
\text { pressure }\end{array}$} \\
\hline$<140 \mathrm{mmHg}$ & $259(72.1)$ & $327(64.5)$ & Ref \\
\hline$\geq 140 \mathrm{mmHg}$ & $100(27.9)$ & $180(35.5)$ & $0.0178^{*}$ \\
\hline \multicolumn{4}{|l|}{$\begin{array}{l}\text { Diastolic blood } \\
\text { pressure }\end{array}$} \\
\hline$<90 \mathrm{mmHg}$ & $281(78.3)$ & $386(75.8)$ & Ref \\
\hline$\geq 90 \mathrm{mmHg}$ & $78(21.7)$ & $123(24.1)$ & 0.4017 \\
\hline \multicolumn{4}{|l|}{ HbA1C (\%) } \\
\hline$<7$ & $141(44.2)$ & $128(27.9)$ & Ref \\
\hline $7-9$ & $109(34.2)$ & $198(43.1)$ & $<0.0001^{* * * *}$ \\
\hline$>9$ & 69 (21.6) & $133(29)$ & $<0.0001^{* * * *}$ \\
\hline \multicolumn{4}{|l|}{$\begin{array}{l}\text { Fasting blood } \\
\text { glucose (mg/dl) }\end{array}$} \\
\hline $70-80$ & $10(3)$ & $12(2.5)$ & Ref \\
\hline $81-120$ & $92(27.6)$ & $119(24.7)$ & 0.8676 \\
\hline $121-160$ & $107(32)$ & $156(32.4)$ & 0.6622 \\
\hline $161-200$ & $71(21.3)$ & $84(17.5)$ & 0.9753 \\
\hline$>200$ & $54(16.2)$ & $110(22.9)$ & 0.2455 \\
\hline \multicolumn{4}{|l|}{$\begin{array}{l}\text { Postprandial blood } \\
\text { glucose levels } \\
(\mathrm{mg} / \mathrm{dl})\end{array}$} \\
\hline $90-110$ & $3(1)$ & $5(1.1)$ & 0.8274 \\
\hline $111-130$ & $9(3)$ & $18(4.1)$ & 0.6043 \\
\hline $131-150$ & $33(10.9)$ & $37(8.4)$ & 0.4425 \\
\hline $151-200$ & $165(54.6)$ & $157(35.8)$ & 0.6155 \\
\hline$>200$ & $92(30.5)$ & $222(50.6)$ & Ref \\
\hline \multicolumn{4}{|l|}{$\begin{array}{l}\text { Random blood } \\
\text { glucose (mg/dl) }\end{array}$} \\
\hline $80-100$ & 0 & $6(5)$ & 0.3359 \\
\hline $101-120$ & 0 & $6(5)$ & 0.3359 \\
\hline $121-140$ & 0 & $6(5)$ & 0.3359 \\
\hline $141-160$ & $2(13.3)$ & $9(7.5)$ & 0.6718 \\
\hline
\end{tabular}


Table 2. (Continued)

\begin{tabular}{|c|c|c|c|}
\hline Variable & $\begin{array}{c}\text { Patients with } \\
\text { T2DM } \\
N(\%)\end{array}$ & $\begin{array}{c}\text { Patients with } \\
\text { T2DM and DPN } \\
N(\%)\end{array}$ & $p$-Value \\
\hline $161-200$ & $1(6.7)$ & $16(13.3)$ & 0.3813 \\
\hline$>200$ & $12(80)$ & $77(64.2)$ & Ref \\
\hline \multicolumn{4}{|l|}{$\mathrm{HDL}(\mathrm{mg} / \mathrm{dl})$} \\
\hline Not available & $54(20.1)$ & $182(38)$ & Ref \\
\hline Normal & $130(48.3)$ & $140(29.2)$ & $<0.0001^{* * *}$ \\
\hline Low & $55(20.4)$ & $110(23)$ & $0.0206^{*}$ \\
\hline High & $30(11.2)$ & $47(9.9)$ & $0.0057^{* * *}$ \\
\hline \multicolumn{4}{|l|}{$\begin{array}{l}\text { Triglycerides } \\
(\mathrm{mg} / \mathrm{dl})\end{array}$} \\
\hline Not available & $54(20.5)$ & $181(37.7)$ & Ref \\
\hline Normal & $109(41.5)$ & $146(30.4)$ & $<0.0001^{* * *}$ \\
\hline Low & $8(3)$ & $12(2.5)$ & 0.0885 \\
\hline High & $92(35)$ & $141(29.4)$ & $0.0001^{* * * *}$ \\
\hline \multicolumn{4}{|l|}{$\begin{array}{l}\text { Total cholesterol } \\
(\mathrm{mg} / \mathrm{dl})\end{array}$} \\
\hline Not available & $54(19.6)$ & $178(37.1)$ & Ref \\
\hline Normal & $151(54.7)$ & $203(42.3)$ & $<0.0001^{* * *}$ \\
\hline Low & $6(2.2)$ & $5(1)$ & $0.0188^{*}$ \\
\hline High & $65(23.6)$ & 94 (19.6) & $0.0002^{* * * *}$ \\
\hline \multicolumn{4}{|l|}{ LDL (mg/dl) } \\
\hline Not available & $57(20.8)$ & $184(38.4)$ & Ref \\
\hline Normal & $163(59.4)$ & $173(36.1)$ & $<0.0001^{* * *}$ \\
\hline Low & $9(3.3)$ & $12(2.5)$ & 0.0518 \\
\hline High & $45(16.5)$ & $110(23)$ & 0.2321 \\
\hline \multicolumn{4}{|l|}{ Urea (mg/dl) } \\
\hline Not available & $72(36.4)$ & $224(50)$ & Ref \\
\hline Normal & $78(39.4)$ & 88 (19.6) & $<0.0001^{* * *}$ \\
\hline Low & 0 & $3(0.7)$ & 0.3269 \\
\hline High & $48(24.2)$ & $133(29.6)$ & 0.5919 \\
\hline \multicolumn{4}{|l|}{$\begin{array}{l}\text { Serum creatinine } \\
(\mathrm{mg} / \mathrm{dl})\end{array}$} \\
\hline Not available & 45 (12.6) & $100(19.6)$ & Ref \\
\hline Normal & $305(85.2)$ & $332(65.2)$ & $0.0002^{* * * *}$ \\
\hline Low & $5(1.4)$ & $3(0.6)$ & 0.0647 \\
\hline High & $3(0.8)$ & 74 (14.6) & $<0.0001^{* * *}$ \\
\hline \multicolumn{4}{|c|}{$\begin{array}{l}\text { Duration of T2DM } \\
\text { (years) }\end{array}$} \\
\hline$<5$ & $172(47.9)$ & $137(27)$ & Ref \\
\hline $5-10$ & $111(30.9)$ & $180(35.4)$ & $<0.0001^{* * *}$ \\
\hline$>10$ & $76(21.2)$ & $191(37.6)$ & $<0.0001^{* * * *}$ \\
\hline \multicolumn{4}{|l|}{$\begin{array}{l}\text { Following T2DM } \\
\text { education }\end{array}$} \\
\hline Yes & $282(79.2)$ & $341(67.5)$ & Ref \\
\hline No & 74 (20.8) & $164(32.5)$ & $0.0002^{* * * *}$ \\
\hline
\end{tabular}

T2DM $=$ type 2 diabetes mellitus, DPN $=$ Diabetic peripheral neuropathy, $\mathrm{BMI}=$ body mass index, HTN = hypertension, $\mathrm{CVDs}=$ cardiovascular diseases, $\mathrm{HbA1C}=$ Glycated hemoglobin, $\mathrm{HDL}=$ high-density lipoproteins, $\mathrm{LDL}=$ low-density lipoproteins.
Table 3. Food and lifestyle characteristics of diabetic patients with $(N=509)$ or without diabetic peripheral neuropathy $(N=359)$.

\begin{tabular}{|c|c|c|c|}
\hline Variable & $\begin{array}{c}\text { Patients with } \\
\text { T2DM } \\
N(\%)\end{array}$ & $\begin{array}{c}\text { Patients with } \\
\text { T2DM and DPN } \\
N(\%)\end{array}$ & $p$-value \\
\hline \multicolumn{4}{|l|}{ Food habits } \\
\hline Vegetarian & $60(16.7)$ & $85(16.7)$ & Ref \\
\hline Mixed & $299(83.3)$ & $424(83.3)$ & 0.9958 \\
\hline \multicolumn{4}{|l|}{ Physical activity } \\
\hline $\begin{array}{l}\text { No physical } \\
\text { activity }\end{array}$ & $176(49)$ & $316(62)$ & Ref \\
\hline Regular exercise & $183(50.9)$ & $193(37.9)$ & $0.0001^{* * * *}$ \\
\hline \multicolumn{4}{|l|}{ Habit of smoking } \\
\hline No & $320(89.1)$ & $442(87)$ & Ref \\
\hline Yes & $22(6.1)$ & $32(6.3)$ & 0.8568 \\
\hline Past smoker & $17(4.7)$ & $34(6.7)$ & 0.2242 \\
\hline \multicolumn{4}{|l|}{$\begin{array}{l}\text { Habit of drinking } \\
\text { alcohol }\end{array}$} \\
\hline No & $304(85.1)$ & $448(88)$ & Ref \\
\hline Yes & $44(12.3)$ & $43(8.4)$ & 0.0689 \\
\hline Past alcoholic & $9(2.5)$ & $18(3.5)$ & 0.4602 \\
\hline \multicolumn{4}{|l|}{$\begin{array}{l}\text { Habit of taking } \\
\text { junk foods }\end{array}$} \\
\hline No & $180(50.3)$ & $187(36.7)$ & Ref \\
\hline Weekly once & $31(8.7)$ & $88(17.3)$ & $<0.0001^{* * * *}$ \\
\hline Weekly twice & $23(6.4)$ & $57(11.2)$ & $0.0010^{* * * *}$ \\
\hline $\begin{array}{l}\text { Weekly thrice } \\
\text { and more }\end{array}$ & $28(7.8)$ & $39(7.7)$ & 0.2743 \\
\hline Occasionally & $96(26.8)$ & $138(27.1)$ & 0.0544 \\
\hline \multicolumn{4}{|l|}{$\begin{array}{l}\text { Habit of taking } \\
\text { fruits/fruit juices }\end{array}$} \\
\hline No & $66(18.5)$ & $116(22.7)$ & Ref \\
\hline Weekly once & $27(7.5)$ & $54(10.6)$ & 0.6463 \\
\hline Weekly twice & $35(9.8)$ & $50(9.8)$ & 0.4406 \\
\hline $\begin{array}{l}\text { Weekly thrice \& } \\
\text { more }\end{array}$ & $125(34.9)$ & $118(23.2)$ & $0.0019^{* *}$ \\
\hline Occasionally & $105(29.3)$ & $171(33.6)$ & 0.7000 \\
\hline \multicolumn{4}{|l|}{$\begin{array}{l}\text { Habit of taking soft } \\
\text { drinks }\end{array}$} \\
\hline No & $272(76.2)$ & $342(67.5)$ & Ref \\
\hline Weekly once & $6(1.7)$ & $11(2.2)$ & 0.4607 \\
\hline Weekly twice & $5(1.4)$ & $11(2.2)$ & 0.2992 \\
\hline $\begin{array}{l}\text { Weekly thrice \& } \\
\text { more }\end{array}$ & $14(4)$ & $8(1.6)$ & 0.0732 \\
\hline Occasionally & $60(16.8)$ & $134(26.4)$ & $0.0010^{* * * *}$ \\
\hline \multicolumn{4}{|l|}{$\begin{array}{l}\text { Habit of taking tea/ } \\
\text { coffee }\end{array}$} \\
\hline No & $55(15.3)$ & 64 (12.6) & Ref \\
\hline $\begin{array}{l}\text { Daily once } \\
\text { without sugar }\end{array}$ & $54(15)$ & $82(16.1)$ & 0.2943 \\
\hline $\begin{array}{l}\text { Daily twice } \\
\text { without sugar }\end{array}$ & $110(30.6)$ & $191(37.5)$ & 0.0674 \\
\hline
\end{tabular}

(Continued) 
Table 3. (Continued)

\begin{tabular}{lccc}
\hline Variable & $\begin{array}{c}\text { Patients with } \\
\text { T2DM } \\
\boldsymbol{N}(\%)\end{array}$ & $\begin{array}{c}\text { Patients with } \\
\text { T2DM and DPN } \\
\boldsymbol{N}(\%)\end{array}$ & $p$-value \\
\hline $\begin{array}{l}\text { Daily thrice } \\
\text { without sugar }\end{array}$ & $58(16.2)$ & $93(18.3)$ & 0.1966 \\
$\begin{array}{l}\text { Daily once with } \\
\text { sugar }\end{array}$ & $25(6.9)$ & $23(4.5)$ & 0.4923 \\
$\begin{array}{l}\text { Daily twice with } \\
\text { sugar }\end{array}$ & $37(10.3)$ & $36(7.1)$ & 0.5476 \\
$\begin{array}{l}\text { Daily thrice with } \\
\text { sugar }\end{array}$ & $20(5.6)$ & $20(3.9)$ & 0.6785 \\
$\begin{array}{l}\text { Situations at } \\
\text { working places }\end{array}$ & $181(50.4)$ & $261(51.3)$ & Ref \\
No stress & $178(49.6)$ & $248(48.7)$ & 0.8031 \\
\hline Stress & & & \\
\hline
\end{tabular}

DPN $=$ Diabetic peripheral neuropathy.

Table 4. Multivariate regression analysis of modifiable and non-modifiable risk factors for the development of diabetic peripheral neuropathy in patients with type 2 diabetes mellitus.

\begin{tabular}{|c|c|c|}
\hline Variable & OR $(95 \% \mathrm{CI})$ & $p$-value \\
\hline \multicolumn{3}{|l|}{ Gender } \\
\hline Male & 1 & Ref \\
\hline Female & $1.184(0.8995-1.557)$ & 0.2284 \\
\hline \multicolumn{3}{|l|}{ Age } \\
\hline $0-20$ years & 1 & Ref \\
\hline $21-40$ years & $1.491(0.05940-37.43)$ & 0.4830 \\
\hline $41-60$ years & $4.393(0.1780-108.4)$ & 0.2267 \\
\hline Above 60 years & $7.852(0.3151-195.7)$ & 0.1070 \\
\hline \multicolumn{3}{|l|}{ Marital status } \\
\hline Unmarried & 1 & Ref \\
\hline Married & $7.868(2.275-27.22)$ & $0.0001^{* * * *}$ \\
\hline \multicolumn{3}{|l|}{ Education } \\
\hline Uneducated & 1 & Ref \\
\hline Educated & $0.2856(0.2151-0.3791)$ & $<0.0001^{* * * *}$ \\
\hline \multicolumn{3}{|l|}{ BMI $\left(\mathrm{Kg} / \mathrm{m}^{2}\right)$} \\
\hline$<25 \mathrm{~kg} / \mathrm{m}^{2}$ & 1 & Ref \\
\hline$\geq 25 \mathrm{Kg} / \mathrm{m}^{2}$ & $1.329(0.9866-1.790)$ & 0.0609 \\
\hline \multicolumn{3}{|l|}{ Body weight $(\mathrm{kg})$} \\
\hline$<50$ & 1 & Ref \\
\hline $50-70$ & $0.5324(0.1880-1.507)$ & 0.2281 \\
\hline$>70$ & $0.4725(0.1673-1.335)$ & 0.1483 \\
\hline \multicolumn{3}{|l|}{ Nature of work } \\
\hline Not working anywhere & 1 & Ref \\
\hline Private job & $0.4219(0.2689-0.6618)$ & $0.0001^{* * * *}$ \\
\hline Govt. job & $0.2175(0.1169-0.4047)$ & $<0.0001^{* * * *}$ \\
\hline Daily labor & $0.3534(0.1991-0.6273)$ & $0.0003^{* * * *}$ \\
\hline Housewife & $0.5254(0.3477-0.7938)$ & $0.0021^{* *}$ \\
\hline \multicolumn{3}{|l|}{ Locality } \\
\hline Rural & 1 & Ref \\
\hline Urban & $0.6602(0.4944-0.8815)$ & $0.0048^{* *}$ \\
\hline \multicolumn{3}{|l|}{ Monthly income } \\
\hline No income & 1 & Ref \\
\hline
\end{tabular}

Table 4. (Continued)

\begin{tabular}{|c|c|c|}
\hline Variable & OR $(95 \% \mathrm{CI})$ & $p$-value \\
\hline Below 25,000 & $0.7462(0.5506-1.011)$ & 0.0587 \\
\hline Above 25,000 & $0.2514(0.1606-0.3933)$ & $<0.0001 * * *$ \\
\hline \multicolumn{3}{|l|}{ Co-morbidities } \\
\hline No & 1 & Ref \\
\hline HTN & $2.642(1.906-3.661)$ & $<0.0001^{* * * *}$ \\
\hline History of CVDs & $10.59(4.648-24.14)$ & $<0.0001^{* * *}$ \\
\hline Endocrine diseases & $1.780(1.170-2.708)$ & $0.0068^{* * *}$ \\
\hline Other diseases & $2.030(1.434-2.874)$ & $<0.0001^{* * * *}$ \\
\hline \multicolumn{3}{|l|}{ Systolic blood pressure } \\
\hline$<140 \mathrm{mmHg}$ & 1 & Ref \\
\hline$\geq 140 \mathrm{mmHg}$ & $1.426(1.063-1.913)$ & $0.0178^{*}$ \\
\hline \multicolumn{3}{|l|}{ Diastolic blood pressure } \\
\hline$<90 \mathrm{mmHg}$ & 1 & Ref \\
\hline$\geq 90 \mathrm{mmHg}$ & $1.148(0.8313-1.585)$ & 0.4017 \\
\hline \multicolumn{3}{|l|}{$\operatorname{HbA1C}(\%)$} \\
\hline$<7$ & 1 & Ref \\
\hline $7-9$ & $2.001(1.432-2.796)$ & $<0.0001^{* * *}$ \\
\hline$>9$ & $2.123(1.457-3.095)$ & $<0.0001^{* * *}$ \\
\hline \multicolumn{3}{|l|}{$\begin{array}{l}\text { Fasting blood glucose } \\
(\mathrm{mg} / \mathrm{dl})\end{array}$} \\
\hline $70-80$ & 1 & Ref \\
\hline $80-120$ & $1.078(0.4460-2.605)$ & 0.8676 \\
\hline $121-160$ & $1.215(0.5066-2.914)$ & 0.6622 \\
\hline $161-200$ & $0.9859(0.4021-2.418)$ & 0.9753 \\
\hline$>200$ & $1.698(0.6899-4.177)$ & 0.2455 \\
\hline \multicolumn{3}{|c|}{$\begin{array}{l}\text { Postprandial blood glucose } \\
\text { levels (mg/dl) }\end{array}$} \\
\hline $90-110$ & 1 & Ref \\
\hline $111-130$ & $1.200(0.2327-6.188)$ & 0.8274 \\
\hline $131-150$ & $0.6727(0.1491-3.035)$ & 0.6043 \\
\hline $151-200$ & $0.5709(0.1341-2.430)$ & 0.4425 \\
\hline$>200$ & $1.448(0.3389-6.186)$ & 0.6155 \\
\hline \multicolumn{3}{|l|}{$\begin{array}{l}\text { Random blood glucose } \\
(\mathrm{mg} / \mathrm{dl})\end{array}$} \\
\hline $80-100$ & $2.097(0.1110-39.60)$ & 0.3359 \\
\hline $101-120$ & $2.097(0.1110-39.60)$ & 0.3359 \\
\hline $121-140$ & $2.097(0.1110-39.60)$ & 0.3359 \\
\hline $141-160$ & $0.7013(0.1348-3.648)$ & 0.6718 \\
\hline $161-200$ & $2.494(0.3022-20.58)$ & 0.3813 \\
\hline$>200$ & 1 & Ref \\
\hline \multicolumn{3}{|l|}{$\operatorname{HDL}(\mathrm{mg} / \mathrm{dl})$} \\
\hline Not available & 1 & Ref \\
\hline Normal & $0.3195(0.2171-0.4702)$ & $<0.0001^{* * *}$ \\
\hline Low & $0.5934(0.3807-0.9251)$ & $0.0206^{*}$ \\
\hline High & $0.4648(0.2683-0.8054)$ & $0.0057^{* * *}$ \\
\hline \multicolumn{3}{|l|}{ Triglycerides (mg/dl) } \\
\hline Not available & 1 & Ref \\
\hline Normal & $0.3996(0.2699-0.5917)$ & $<0.0001^{* * *}$ \\
\hline Low & $0.4475(0.1739-1.151)$ & 0.0885 \\
\hline High & $0.4572(0.3059-0.6834)$ & $0.0001^{* * *}$ \\
\hline
\end{tabular}


Table 4. (Continued)

\begin{tabular}{|c|c|c|}
\hline Variable & OR $(95 \% \mathrm{CI})$ & $p$-value \\
\hline \multicolumn{3}{|l|}{ Total cholesterol (mg/dl) } \\
\hline Not available & 1 & Ref \\
\hline Normal & $0.4078(0.2816-0.5906)$ & $<0.0001^{* * * *}$ \\
\hline Low & $0.2528(0.07422-0.8611)$ & $0.0188^{*}$ \\
\hline High & $0.4387(0.2828-0.6806)$ & $0.0002^{* * * *}$ \\
\hline \multicolumn{3}{|l|}{$\mathrm{LDL}(\mathrm{mg} / \mathrm{dl})$} \\
\hline Not available & 1 & Ref \\
\hline Normal & $0.3288(0.2280-0.4742)$ & $<0.0001^{* * * *}$ \\
\hline Low & $0.4130(0.1656-1.030)$ & 0.0518 \\
\hline High & $0.7572(0.4796-1.196)$ & 0.2321 \\
\hline \multicolumn{3}{|l|}{ Urea (mg/dl) } \\
\hline Not available & 1 & Ref \\
\hline Normal & $0.3626(0.2420-0.5433)$ & $<0.0001^{* * * *}$ \\
\hline Low & $2.261(0.1153-44.32)$ & 0.3269 \\
\hline High & $0.8906(0.5830-1.360)$ & 0.5919 \\
\hline \multicolumn{3}{|l|}{ Serum creatinine(mg/dl) } \\
\hline Not available & 1 & Ref \\
\hline Normal & $0.4898(0.3334-0.7197)$ & $0.0002^{* * *}$ \\
\hline Low & $0.2700(0.06181-1.179)$ & 0.0647 \\
\hline High & $11.10(3.320-37.11)$ & $<0.0001^{* * * *}$ \\
\hline \multicolumn{3}{|l|}{ Duration of T2DM (years) } \\
\hline$<5$ & 1 & Ref \\
\hline $5-10$ & $2.036(1.469-2.821)$ & $<0.0001^{* * * *}$ \\
\hline$>10$ & $3.155(2.228-4.468)$ & $<0.0001^{* * *}$ \\
\hline \multicolumn{3}{|l|}{$\begin{array}{l}\text { Following T2DM } \\
\text { education }\end{array}$} \\
\hline Yes & 1 & Ref \\
\hline No & $1.833(1.335-2.516)$ & $0.0002^{* * * *}$ \\
\hline \multicolumn{3}{|l|}{ Food habits } \\
\hline Vegetarian & 1 & Ref \\
\hline Mixed & $1.001(0.6968-1.438)$ & 0.9958 \\
\hline \multicolumn{3}{|l|}{ Physical activity } \\
\hline No physical activity & 1 & Ref \\
\hline Regular exercise & $0.5874(0.4467-0.7723)$ & $0.0001^{* * * *}$ \\
\hline \multicolumn{3}{|l|}{ Habit of smoking } \\
\hline No & 1 & Ref \\
\hline Yes & $1.053(0.6005-1.847)$ & 0.8568 \\
\hline Past smoker & $1.448(0.7948-2.638)$ & 0.2242 \\
\hline \multicolumn{3}{|l|}{ Habit of drinking alcohol } \\
\hline No & 1 & Ref \\
\hline Yes & $0.6631(0.4250-1.035)$ & 0.0689 \\
\hline Past alcoholic & $1.357(0.6016-3.061)$ & 0.4602 \\
\hline \multicolumn{3}{|l|}{ Habit of taking junk foods } \\
\hline No & 1 & Ref \\
\hline Weakly once & $2.732(1.729-4.319)$ & $<0.0001^{* * * *}$ \\
\hline Weekly twice & $2.385(1.410-4.036)$ & $0.0010^{* * * *}$ \\
\hline Weekly thrice and more & $1.341(0.7915-2.271)$ & 0.2743 \\
\hline Occasionally & $1.384(0.9934-1.927)$ & 0.0544 \\
\hline
\end{tabular}

(Continued)
Table 4. (Continued)

\begin{tabular}{|c|c|c|}
\hline Variable & OR $(95 \% \mathrm{CI})$ & $p$-value \\
\hline \multicolumn{3}{|l|}{$\begin{array}{l}\text { Habit of taking fruits/fruit } \\
\text { juices }\end{array}$} \\
\hline No & 1 & Ref \\
\hline Weekly once & $1.138(0.6551-1.977)$ & 0.6463 \\
\hline Weekly twice & $0.8128(0.4797-1.377)$ & 0.4406 \\
\hline Weekly thrice \& more & $0.5371(0.3625-0.7959)$ & $0.0019^{* *}$ \\
\hline Occasionally & $0.9266(0.6287-1.366)$ & 0.7000 \\
\hline \multicolumn{3}{|l|}{ Habit of taking soft drinks } \\
\hline No & 1 & Ref \\
\hline Weekly once & $1.458(0.5323-3.994)$ & 0.4607 \\
\hline Weekly twice & $1.750(0.6006-5.098)$ & 0.2992 \\
\hline Weekly thrice \& more & $0.4545(0.1879-1.099)$ & 0.0732 \\
\hline Occasionally & $1.776(1.260-2.505)$ & $0.0010^{* * * *}$ \\
\hline \multicolumn{3}{|l|}{ Habit of taking tea/coffee } \\
\hline No & 1 & Ref \\
\hline $\begin{array}{l}\text { Daily once without } \\
\text { sugar }\end{array}$ & $1.305(0.7931-2.147)$ & 0.2943 \\
\hline $\begin{array}{l}\text { Daily twice without } \\
\text { sugar }\end{array}$ & $1.492(0.9706-2.294)$ & 0.0674 \\
\hline $\begin{array}{l}\text { Daily thrice without } \\
\text { sugar }\end{array}$ & $1.378(0.8464-2.243)$ & 0.1966 \\
\hline Daily once with sugar & $0.7906(0.4040-1.547)$ & 0.4923 \\
\hline Daily twice with sugar & $0.8361(0.4665-1.499)$ & 0.5476 \\
\hline Daily thrice with sugar & $0.8594(0.4195-1.760)$ & 0.6785 \\
\hline \multicolumn{3}{|l|}{$\begin{array}{l}\text { Situations at working } \\
\text { places }\end{array}$} \\
\hline No stress & 1 & Ref \\
\hline Stress & $0.9662(0.7374-1.266)$ & 0.8031 \\
\hline
\end{tabular}

T2DM = type 2 diabetes mellitus, BMI = body mass index, HTN = hypertension, $\mathrm{CVDs}=$ cardiovascular diseases, $\mathrm{HbA1C}=$ glycated hemoglobin, $\mathrm{HDL}=$ high-density lipoproteins, $\mathrm{LDL}=$ low-density lipoproteins.

Education is one of the risk factors for the development of DPN. Kiani et al. (2013) conducted a study on the prevalence and associated risk factors of DPN in Hamedan, Iran, and concluded that education qualification was significantly related to the presence of diabetic neuropathy (Kiani et al., 2013). In this study, educational status was associated with DPN (66.8\%, $p<0.0001)$ and a risk factor for the development of DPN. In the current study, housewives $(43.2 \%, p=0.0021)$ were associated with DPN and they are at high risk for DPN (OR, 0.5254; $95 \%$ CI, 0.3477-0.7938). Previous reports are not available on this variable.

The present study results revealed that urban residents $(61.5 \%, p=0.0048)$ were significantly associated and are at high risk for DPN (OR, 0.6602; 95\% CI, 0.4944-0.8815). Previous reports are not available on this variable. Hypertension $(p<0.0001)$ was positively associated with DPN. Dipika et al., (2014) conducted a study on prevalence and risk factors of development of DPN in T2DM in a tertiary care setting and concluded that hypertension was found significant for neuropathy in T2DM patients (Dipika et al., 2014). In the current study also hypertension $(40.41 \%, p<0.0001)$ was a risk factor for DPN (OR, 2.642 ; $95 \%$ CI, $1.906-3.661$ ). 
Table 5. Medication given for the patients with diabetic peripheral neuropathy.

\begin{tabular}{|c|c|c|}
\hline S. No & Generic name of drugs & $N(\%)$ \\
\hline 1 & Metformin & $119(40.47)$ \\
\hline 2 & Glimepiride + Metformin & $88(29.93)$ \\
\hline 3 & Isophane Insulin + Regular Insulin & $67(22.7)$ \\
\hline 4 & Glimepiride & $27(9.18)$ \\
\hline 5 & Gliclazide + Metformin & $25(8.50)$ \\
\hline 6 & Insulin Glargine & $21(7.14)$ \\
\hline 7 & Insulin Aspart & $17(5.78)$ \\
\hline 8 & Pioglitazone & $15(5.10)$ \\
\hline 9 & Teneligliptin & $13(4.4)$ \\
\hline 10 & Gliclazide & $12(4.08)$ \\
\hline 11 & Sitagliptin + Metformin & $10(3.40)$ \\
\hline 12 & Insulin Lispro + Protamine Insulin & $10(3.40)$ \\
\hline 13 & Teneligliptin + Metformin & $6(2.04)$ \\
\hline 14 & Glipizide + Metformin & $6(2.04)$ \\
\hline 15 & Voglibose & $6(2.04)$ \\
\hline 16 & Acarbose & $5(1.70)$ \\
\hline 17 & Insulin Aspart + Protamine Insulin & $5(1.70)$ \\
\hline 18 & Sitagliptin & $4(1.36)$ \\
\hline 19 & Metformin + Vildagliptin & $4(1.36)$ \\
\hline 20 & Pioglitazone + Metformin & $4(1.36)$ \\
\hline 21 & Dapagliflozin & $3(1.02)$ \\
\hline 22 & Metformin + Voglibose & $3(1.02)$ \\
\hline 23 & Empagliflozin & $2(0.68)$ \\
\hline 24 & Vildagliptin & $2(0.68)$ \\
\hline 25 & Glipizide & $2(0.68)$ \\
\hline 26 & Repaglinide & $2(0.68)$ \\
\hline 27 & Glibenclamide + Metformin & $2(0.68)$ \\
\hline 28 & Canagliflozin & $1(0.34)$ \\
\hline 29 & Linagliptin & $1(0.34)$ \\
\hline 30 & Insulin Degludec + Insulin Aspart & $1(0.34)$ \\
\hline 31 & Lantus Insulin & $1(0.34)$ \\
\hline 32 & Acarbose + Metformin & $1(0.34)$ \\
\hline 33 & Linagliptin + Metformin & $1(0.34)$ \\
\hline 34 & Glimepiride + Metformin + Voglibose & $1(0.34)$ \\
\hline 35 & Glimepiride + Metformin + Pioglitazone & $1(0.34)$ \\
\hline
\end{tabular}

Harry et al. (2017) conducted a study on risk factors for neuropathic pain in diabetes mellitus and concluded that physical inactivity was found significant for neuropathy in T2DM patients. The present study results also revealed that physical inactivity $(62 \%, p<0.0001)$ was associated and is the major risk factor for DPN. The present study results revealed that habit of taking junk foods weekly once $(17.3 \%, p<0.0001)$, weekly twice $(11.2 \%$, $p=0.0010)$ was significantly associated and are the major risk factor for DPN (weekly once OR, 2.732; 95\% CI, 1.729-4.319) and weekly twice (OR, 2.385; 95\% CI, 1.4102-4.036).

The present study results revealed that the habit of taking soft drinks occasionally $(26.4 \%, p=0.0010)$ was associated and was the major risk factor for DPN (OR, 1.776; 95\% CI, 1.260-2.505). Therefore, further studies are needed to evaluate the exact impact of the habit of taking junk foods on risk for DPN. Poor glycemic control was significantly associated with the development of DPN. A study conducted by Dong et al. on the prevalence of DPN in a Saudi Population and concluded that duration of diabetes and glycemic control were strongly associated with DPN (Wang et al., 2014). In this study also poor glycemic control $(43.1 \%, p<0.0001)$ was a risk factor for the development of DPN (OR, 2.001; 95\% CI, 1.432-2.796). Another study was conducted by Muhammad et al., (2015) on the association of DPN with duration of T2DM and glycemic control and concluded that DPN was a problematic complication of diabetes.,. Our findings are also in the same line with the findings of Jiali et al. (2015) and Dipika et al. (2014).

Harry et al. (2017) conducted a study on risk factors for neuropathic pain in diabetes mellitus and concluded that low HDL levels were found significant for neuropathy in T2DM patients. The present study results revealed that low HDL levels $(23 \%$, $p=0.0206)$ were significantly associated and were the major risk factor for DPN (OR, 0.5934; 95\% CI, 0.3807-0.9251). Wang et al. (2014) conducted a study on the prevalence of DPN and concluded that a novel clinical marker (creatinine) was also identified, which may contribute to the risk prediction of DPN. In the current study also high serum creatinine levels $(14.6 \%, p<0.0001)$ was a risk factor for DPN (OR, 11.10; 95\% CI, 3.320-37.11).

Monisha et al., (2015) conducted a study on DPN and concluded that the burden of DPN is high among diabetics with a long duration of T2DM. These results were supported by the present study $(37.6 \%$, OR, 3.155; 95\% CI, 2.228-4.468). Other relevant studies conducted by Muhammad et al. (2015), Kiani et al. (2013), and Jiali (2015) also concluded that long duration of diabetes significantly associated with DPN.

Sekhar et al. (2016) conducted a study and identified that metformin was the widely used drug followed by glimepiride and metformin combination). Our present study results also revealed that metformin was the widely used drug followed by glimepiride and metformin combination.

\section{CONCLUSION}

The present study revealed that hypertension, endocrine diseases, history of CVDs, poor glycemic control ( $>9 \mathrm{HbAlc}$ ), low HDL, high serum creatinine, long duration of diabetes, physical inactivity, habit of taking junk foods and soft drinks were the significant risk factors for the development of DPN. Early detection of the identification of DPN in T2DM is needed in order to slow progression and complications. Metformin (40.47\%), combination of glimepiride and metformin (29.93\%), combination of human insulin and insulin isophane $(22.7 \%)$ were mostly given to the T2DM patients with neuropathy.

\section{ACKNOWLEDGMENTS}

The authors would like to thank the management (Siddhartha Academy of General and Technical Education) of KVSR SCOPS for their support in the completion of this work. The authors also thank Dr. Jaidev Sudagani, consultant endocrinologist and diabetologist, Santhi Endocrine and Diabetes hospital for providing the necessary facilities.

\section{CONFLICT OF INTEREST}

The authors declared that they have no conflict of interests. 


\section{SOURCE OF FUNDING}

None.

\section{REFERENCES}

Amit S, Pooja S, Ankit G, Manik C, Rupinderjeet K. A crosssectional study on diabetes mellitus type-2 at a tertiary care hospital. Adv Res Gastroentero Hepatol, 2017; 8(1):555726; doi:10.19080/ ARGH.2017.08.555726

Boulton AJ, Gries FA, Jervell JA. Guidelines for the diagnosis and outpatient management diabetic peripheral neuropathy. Diabet Med, 1998; 15(6):508-14.

Bruce SG, Young TK. Prevalence and risk factors for neuropathy in a Canadian First Nation Community. Diabetes Care, 2008; 31:1837-41.

Candrilli SD, Davis KL, Kan HJ, Lucero MA, Rousculp M. Prevalence and the associated burden of illness of symptoms of diabetic peripheral neuropathy and diabetic retinopathy. J Diabetes Complications, 2007; 21(5):306-14.

Davies M, Brophy S, Williams R, Taylor A. The prevalence, severity, and impact of painful diabetic peripheral neuropathy in type 2 diabetes. Diabetes Care, 2006; 29:1518-22.

Dipika B, Kapil G, Harini M, Hari PE, Ramya N, Anil B. Prevalence and risk factors of development of peripheral diabetic neuropathy in type 2 diabetes mellitus in a tertiary care setting. J Diabetes Investig, 2014; 5(6):714-21.

Emanuele NV, Swade TF, Emanuele MA. Consequences of alcohol use in diabetics. Alcohol Health Res World, 1998; 22:211-9.

Harry LH, Abirami V, Nicola T, Blair HS. Risk factors for neuropathic pain in diabetes mellitus. Pain, 2017; 158:560-8.

Jiali C. Prevalence and risk factors of diabetic peripheral neuropathy in type 2 diabetes mellitus (T2DM) patients. Prim Care Diabetes, 2015; 9(3):191-5.

Joaoda RF, Katherine O, Ute Lk, Leonor G, Till S, Ping Z, David C, Lydia EM. IDF Diabetes Atlas estimates of 2014 global health expenditures on diabetes. Diabetes Res Clin Pract, 2016; 117:48-54.

Kiani J, Moghimbeigi A, Azizkhani H, Kosarifard S. The prevalence and associated risk factors of peripheral diabetic neuropathy in hamedan, iran. Arch Iran Med, 2013; 16(1):17-9.

Monisha DS, Vaman K, Unnikrishnan B, Humam A, Hegde N, Anjali P, Tabreez S, Bhanu D, Rekha T, Prasanna M, Nithin K, Ramesh H, Darshan BB, and Avinash K Diabetic. Peripheral neuropathy and its determinants among patients attending a tertiary health care centre in Mangalore. Indian J Public Health Res, 2015; 4(2):450.

Muhammad UN, Ambreen A, Ahmed W, Nazia A, Anam N, Mohsin AQ, Hafsa M, Mohsin J, Mohsin J. Association of diabetic neuropathy with duration of type 2 diabetes and glycemic control. Cureus, 2015; 7(8):e302.
Nahla K, Jawad AS, Mohammed S, Said SD, Yousef SK and Kamel MA. The prevalence and risk factors of peripheral neuropathy among patients with type 2 diabetes mellitus; the case of Jordan. Diabetol Metab Syndr, 2018; 10(8):1-10; doi:10.1186/s13098-018-0309-6

Sekhar M, Tamoghna M, Asoke KD, Abhijit D, Ananya M, Biswanath SS, Soumitra M. Drug utilization study in patients with type 2 diabetes mellitus attending diabetes clinic of a tertiary care hospital in rural Bengal. IJBCP, 2016; 5(4):1647.

Sumner CJ, Sheth S, Griffin JW, Cornblath DR, Polydefkis M. The spectrum of neuropathy in diabetes and impaired glucose tolerance. Neurology, 2003; 60(1):108-11.

Tesfaye S. Epidemiology and etiology of diabetic peripheral neuropathies. Adv Stud Med, 2004; 4:S1014-21.

Tesfaye S, Selvarajah D. Advances in the epidemiology, pathogenesis and management of diabetic peripheral neuropathy. Diabetes Metab Res Rev, 2012; 28:8-14.

Tesfaye S, Stevens LK, Stephenson JM, Fuller JH, Plater M, Ionescu-Tirgoviste C, Nuber A, Pozza G, Ward JD. Prevalence of diabetic peripheral neuropathy and its relation to glycaemic control and potential risk factors: the EURODIAB IDDM Complications Study. Diabetologia, 1996; 39:1377-84.

Wang DD, Bakhotmah BA, Hu FB, Alzahrani HA. Prevalence and correlates of diabetic peripheral neuropathy in a saudi arabic population: a cross-sectional study. PLoS One, 2014; 9(9):e106935.

William HH, Laurence K. Underdiagnosis of peripheral neuropathy in type 2 diabetes. Diabetes Care, 2005; 28(6):1480-1.

Young M, Boulton A, MacLeod A, Williams D, Sonksen P. A multicentre study of the prevalence of diabetic peripheral neuropathy in the United Kingdom hospital clinic population. Diabetologia, 1993; 36:150-4.

How to cite this article:

Nalluri L, Mannam M, Vemireddy N, Akula N, Pothuraju M, Pinnika D, Sudagani J, Pingili R, Kilaru NB. Assessment of drug utilization pattern and risk factors for the development of diabetic neuropathy among type 2 diabetic patients in a south Indian hospital: a cross-sectional observational study. J Appl Pharm Sci, 2019; 9(12):069-077. 